\title{
CHAPTER 5B
}

\section{COMMENTS ON "FORAGING IN A HETEROGENEOUS ENVIRONMENT: INTAKE AND DIET CHOICE”}

\author{
MICHAEL DRESCHER ${ }^{\#}$, HERBERT H.T. PRINS \#\# AND \\ AREND M.H. BRUNSTING \#\#

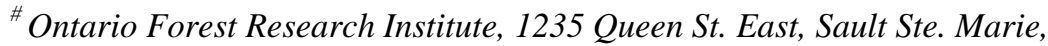 \\ Ontario, P6A 2E5, Canada \\ ${ }^{\#}$ Resource Ecology Group, Wageningen University, P.O. Box 47, \\ 6700 AA Wageningen, The Netherlands \\ E-mail: michael.drescher@mnr.gov.on.ca
}

In Chapter 5, Laca poses that currently there is no general quantitative theory of large-herbivore foraging behaviour in landscapes with heterogeneous resources. Though his stated goal here is not to put forward such a theory, he aims to present a number of relevant concepts and theories, and to place them in a coherent framework. Based on these, he poses some questions and hypotheses in an attempt to fill apparent knowledge gaps.

Laca starts his chapter by giving a general description of some concepts of spatial heterogeneity of vegetation. He explains how herbivore size and mobility can determine an animal's perception of heterogeneity, and the minimal and maximal scales on which an animal may respond to it. Then, he argues that the multidimensional character of heterogeneity can be reduced to a very limited number of dimensions that need to be understood in order to sufficiently describe herbivore foraging behaviour. He continues by illustrating mechanisms through which forage resource heterogeneity affects forage intake and diet selection. The spatial scales of the experiments he uses to support his points vary from a single feeding station to pastures of several hectares. Most of these studies investigate the effects of grass height on intake in mono-specific swards, and of proportion and spatial arrangement of two qualitatively different forage species on diet choice and intake.

101

Herbert H.T. Prins, Frank van Langevelde (eds.), Resource Ecology: Spatial and Temporal Dynamics of Foraging, 101-103.

(C) 2008 Springer. 
Perception and mobility implicitly are quite central to Laca's coverage of the effects of heterogeneity on forage intake and diet choice. Perception essentially sets a lower limit, while mobility sets an upper limit to the heterogeneity that a herbivore can respond to. Laca is touching upon these points in Box 5.1 with his example of the rabbit and the bison. However, he largely misses the opportunity to stress clearly that perception and mobility are likely affected by animal size and thus speciesspecific.

In Laca's example of the mixed swards of grass and clover (Figure 5.11), smaller herbivores like sheep can still select a higher-quality diet. But a large herbivore whose ingestive apparatus is several times the size of the distance between the clusters of clover, would not be able to select the clover from in-between the grass. In such an arrangement of things, a large herbivore's perception of the spatial variation in forage quality between clover and grass would be of no advantage. Therefore, we propose that it is unlikely that a large forager will perceive heterogeneity at a very small scale. An example of the varying selection ability between differently sized herbivores is browsing kudu, which are able to select small clusters of high-quality leaves while black rhino browse whole branches (Wilson and Kerley 2003).

Laca's example of the rabbit and the bison in Box 5.1 is also an illustration of the effects of body size on mobility and its subsequent effect on the perception of largescale spatial variation in resource heterogeneity. Generally, smaller herbivores are likely to have smaller territories and home ranges than larger herbivores (Mysterud et al. 2001). This may not only be the result of reduced travelling speed in smaller herbivores, but also due to their increased need for cover, which is modified in some species by their tendency to congregate in larger herds. The perception of resource heterogeneity above the spatial scale of the territory or home ranges would be of no advantage to a herbivore. As there is no advantage from the perception of this heterogeneity, we propose that it is unlikely that a herbivore has developed a sensory system to perceive heterogeneity at that scale. Real-world examples for the described differences in home ranges and response to resource heterogeneity can be found for many antelope species of different size (Garland et al. 1993), where smaller antelopes tend to stay put during the dry season while larger antelopes may follow the rain (Fryxell, Chapter 6; Fryxell et al. 2005).

Treating a forage resource as a surface, as done by Emilio Laca, might be appropriate in the situation of herbivores grazing on pastures or short-grass savannas, where sward bulk density is high or distances between plants are small. However, forage resources actually exist in 3-dimenional space, and viewing them as a plain surface might often be an oversimplification. Ruyle et al. (1987) have shown that in some situations cattle graze on grass tufts by approaching them from the side instead from above, apparently to avoid grass stems. Also Drescher (2003) found that free-ranging cattle in tall-grass savannas grazed tall grass tufts from sideways. Laca's concept of the relations between bite size and sward height alone appears too simple to describe forage intake in such grasslands. Instead, as shown by Drescher et al. (2006a) forage accessibility, i.e., the ease with which preferred forage parts can be harvested as affected by the spatial arrangement of plant parts 
increases in importance for understanding grazers' intake. It might be possible to grasp the structural complexity of forage resources in 3 dimensions with a measure of fractal geometry as proposed in Box 5.1 of Chapter 5. However, the success of such an approach is uncertain, as demonstrated by a failed attempt to relate forage intake to fractal geometry of grasslands by Drescher et al. (2006b). It might be that a measure of fractal geometry will not be informative, because structural complexity in natural grasslands co-varies with forage mass, sward height, and many other variables. At least, it will need very careful experimental set-ups to isolate the effect of structural complexity from the effects of other variables.

Another aspect that Laca only refers to in passing but that deserves more attention is resource heterogeneity in the temporal dimension (see also Owen-Smith, Chapter 8). Temporal resource heterogeneity can be brought about by ageing and depletion of the forage resource. However, the majority of studies cited by Laca to illustrate his points do not take these aspects into account. This approach implies that Laca assumes that herbivores can always avoid the negative effects of forage resource depletion by moving between available resources, or that herbivores do not respond to temporal changes in resource abundance and quality. We believe that neither one would be a correct assumption. Though many large herbivores seem to track the spatially and temporally shifting abundance and quality of forage resources, other herbivores do not seem to do this, or to a much lesser degree. In this latter case, one would expect that herbivores show behavioural and physiological changes in response to the temporal heterogeneity in the forage resource. Examples of such behavioural and physiological changes are suggested by studies on seasonal changes in selectivity in goats (Duncan et al. 2005) and rumen fermentation in sheep (Sankhyan et al. 2001).

We do not agree with Laca's statement that "in practical terms heterogeneity impacts will not produce responses that are significantly different from predictions based on the study of homogeneous vegetation”. We believe that Laca himself and also we in this comment illustrate numerous concepts of the effects of resource heterogeneity on large-herbivore foraging behaviour. But it is especially the intermediate scales where knowledge about foraging behaviour is currently lacking. Advances in our understanding can be expected from the field of animal cognition. For example, work about the spatial memory of herbivores has not only shown their great capacities (e.g., Edwards et al. 1996), but also some limits of their abilities (Dumont et al. 2000). We want to close by agreeing with Laca that a general theory of large-herbivore foraging in landscapes of heterogeneous resources is needed to put past and more recent study results into perspective and to generate relevant hypotheses. We hope that Laca's chapter in this volume and our comments will bring us closer to reaching this aim. 